PROCEEDINGS OF THE

AMERICAN MATHEMATICAL SOCIETY

Volume 136, Number 1, January 2008, Pages 287-294

S 0002-9939(07)08941-1

Article electronically published on September 24, 2007

\title{
THE $r$-STABILITY OF HYPERSURFACES WITH ZERO GAUSS-KRONECKER CURVATURE
}

\author{
MARCOS P. A. CAVALCANTE
}

(Communicated by Richard A. Wentworth)

\begin{abstract}
In this paper we give sufficient conditions for a bounded domain in an $r$-minimal hypersurface of the Euclidean space to be $r$-stable. The GaussKronecker curvature of this hypersurface may be zero on a set of capacity zero.
\end{abstract}

\section{INTRODUCTION}

Let $M$ be an oriented hypersurface of the $(n+1)$-dimensional Euclidean space and let $g: M \rightarrow S^{n}$ denote its Gauss map. The shape operator of $M$ is the self-adjoint map given by $B:=-d g$, that is, for each $p \in M$,

$$
B_{p}: T_{p} M \rightarrow T_{p} M, B_{p}(X)=-d g_{p}(X) .
$$

The eigenvalues of $B_{p}$ are called the principal curvatures of $M$ at $p$. We denote them by $k_{1}(p), \ldots, k_{n}(p)$, and we define the $r$-mean curvature of $M$ as the normalized $r$-elementary symmetric function of the principal curvatures of $M$, namely,

$$
H_{0}=1, H_{r}=\left(\begin{array}{l}
n \\
r
\end{array}\right)^{-1} S_{r}, r=1, \ldots, n,
$$

where $S_{r}=\sum_{i_{1}<\ldots<i_{r}} k_{i_{1}} \ldots k_{i_{r}}$. Notice that $H_{1}, H_{2}, H_{n}$ are the mean, scalar and Gauss-Kronecker curvatures of $M$, respectively.

We say that $M$ is $r$-minimal when $H_{r+1}=0$. It is well known that the $r$-minimal hypersurfaces of the Euclidean space are critical points of the $r$-area functional $A_{r}=\int_{M} S_{r} d M$ for compactly supported variations of $M$.

In order to state our results we need more notation. Let $P_{r}$ be the Newton transformations of $B$, which can be defined inductively by

$$
P_{0}=I, P_{r}=S_{r} I-B P_{r-1}, \quad r=1,2, \ldots, n .
$$

Let $C_{0}^{\infty}(M)$ denote the set of smooth functions with compact support on $M$. Using the Newton transformations we define the linear operator

$$
L_{r}(f)=\operatorname{div}\left(P_{r} \nabla f\right), \quad \text { for } f \in C_{0}^{\infty}(M) .
$$

Received by the editors April 20, 2006 and, in revised form, September 22, 2006.

2000 Mathematics Subject Classification. Primary 53C42, 53A07; Secondary 35P15.

Key words and phrases. $r$-minimal immersions, $r$-stability, capacity.

The author was fully supported by CNPq-Brazil.

(C)2007 American Mathematical Society Reverts to public domain 28 years from publication 
We denote by $T_{r}$ the Jacobi operator

$$
T_{r} f=L_{r} f-(r+2) S_{r+2} f
$$

and by $I_{r}\left(f_{1}, f_{2}\right)=-\int_{M} f_{1} T_{r}\left(f_{2}\right) d M$ the associated bilinear symmetric form.

Let $D$ be a regular domain on $M$, that is, $D$ is bounded and has piecewise smooth boundary. Following [1, we say that $D$ is $r$-stable if $I_{r}(f, f) \geq 0$ for all $f \in C_{0}^{\infty}(D)$ or if $I_{r}(f, f) \leq 0$ for all $f \in C_{0}^{\infty}(D)$. Otherwise we say that $D$ is $r$-unstable.

In the study of $r$-stability we need to suppose that $L_{r}$ is elliptic. This is equivalent to $P_{r}$ being positive definite or negative definite everywhere. On the other hand, by a theorem of Hounie-Leite in [7, it is known that, when $H_{r+1}=0$, then $L_{r}$ is elliptic if and only if $\operatorname{rank}(B)>r$. In the following, without loss of generality, we will fix $P_{r}>0$. Also, the eigenvalues of the operator $\sqrt{P_{r}} B$ appear naturally, and we will denote them by $\theta_{1}(r), \theta_{2}(r), \ldots, \theta_{n}(r)$.

In [1 Alencar, do Carmo and Elbert gave sufficient conditions for a regular domain on an $r$-minimal hypersurface of the Euclidean space to be $r$-stable. Their general result assumes that the quotient $\frac{\left|H_{n}\right|}{\left\|\sqrt{P_{r}} B\right\|^{2}}$ is constant. In this case the hypersurface is said to be $r$-special.

Theorem A (Theorem 1.3 of [1 ). Let $x: M^{n} \rightarrow \mathbb{R}^{n+1}$ be an oriented r-special hypersurface with $H_{r+1}=0$ and $H_{n} \neq 0$ everywhere. Let $D \subset M$ be a regular domain such that the area of $g(D) \subset S^{n}$ is smaller than the area of a spherical cap whose first eigenvalue for the spherical Laplacian is

$$
\tau=\max _{i, D}\left(\frac{\sum_{j} \theta_{j}^{2}(r)}{\theta_{i}^{2}(r)}\right) .
$$

Then $D$ is $r$-stable.

This theorem is a generalization of a classical result to minimal surfaces of $\mathbb{R}^{3}$ due to Barbosa and do Carmo in 3. We point out that the hypothesis of $M$ to be $r$-special occurs naturally when $r=n-2$. In fact, a computation shows that $\frac{\left|S_{n}\right|}{\left\|\sqrt{P_{n-2}} B\right\|^{2}}=\frac{1}{n}$.

In this paper we are interested in improving the condition on the Gauss-Kronecker curvature considering points on $M$ for which $H_{n}=0$. A simple example, like a cylinder over a plane curve, shows that $H_{n}$ cannot be identically zero. In [1, remark 4.2, it was conjectured that Theorem $\mathrm{A}$ holds if the set of zeros of $H_{n}$ is contained in a submanifold of codimension $d \geq 2$. Here we answer this conjecture affirmatively as a consequence of a more general result. We will consider hypersurfaces with $H_{n}=0$ on a subset of capacity zero (see below the definition of capacity). It is known (see $8 \S 2$, p. 35) that submanifolds of codimension $d \geq 2$ have capacity zero. In section 2 we will give definitions and develop some facts about capacity. To state our result we will denote by A the set $A=\left\{p \in M: H_{n}(p)=0\right\}$ and by $\lambda_{1}(D)$ the first eigenvalue for the Laplacian on $D$. We also point out that, since symmetrization of domains in the sphere does not increase eigenvalues, the hypothesis on the first eigenvalue of the spherical image of $D$ in Theorem $\mathrm{A}$ implies that $\lambda_{1}(g(D)) \geq \tau$. For simplicity we will use this condition on our result below.

Theorem 1.1. Let $M$ be an oriented $r$-minimal hypersurface of $\mathbb{R}^{n+1}$, which is $r$-special on $M \backslash A$. Let $D \subset M$ be a regular domain such that $\lambda_{1}(g(D)) \geq \tau$. Then, if $\operatorname{Cap}(D \cap A)=0, D$ is r-stable. 
The idea of the proof is to use a relation between the eigenvalues of domains from which we remove a subset and the capacity of the removed subset. Actually, we need just a comparison between the first eigenvalues of $P_{r}$ on $D$ and on $D \backslash A$. This relation is well known for the Laplacian and we can find it in [8], for domains of the Euclidean space, and in 4, for domains of a closed Riemannian manifold. Here we obtain such results for an elliptic operator $L$ in divergence form on a bounded domain $D$ of an $n$-dimensional Riemannian manifold $M$. Given $A \subset D$, let $\lambda_{k}(D)$ and $\lambda_{k}(D \backslash A)$ denote the $k$-th eigenvalue of the Dirichlet problem of $L$ on $D$ and on $D \backslash A$, respectively. We have the following result that will be proved in the next section

Theorem 1.2. For an elliptic operator $L$ in divergence form there exist positive constants $\varepsilon_{k}$ and $C_{k}$, such that if $\operatorname{Cap} A \leq \varepsilon_{k}$, then

$$
\lambda_{k}(D) \leq \lambda_{k}(D \backslash A) \leq \lambda_{k}(D)+C_{k} \operatorname{Cap} A^{\frac{1}{2}} .
$$

In particular, $\lambda_{k}(D)=\lambda_{k}(D \backslash A)$ if Cap $A=0$.

\section{The SPECTRUM OF DOMAINS AND THE CAPACITY}

In this section we introduce the notion of capacity and recall some spectral properties of domains in Riemannian manifolds. In this section we also prove Theorem 1.2 .

Let $\left(M^{n},\langle\rangle,\right)$ be a smooth Riemannian manifold and $D \subset M$ a bounded domain. As usual, we define $H_{0}^{1}(D)$ as the closure of $C_{0}^{\infty}(D)$ with respect to the norm $H^{1}$ :

$$
|u|_{H^{1}}^{2}:=\int_{D} u^{2} d M+\int_{D}|\nabla u|^{2} d M, \text { for } u \in C_{0}^{\infty}(D)
$$

where $\nabla$ is the gradient, $|$.$| is the norm of a vector, and d M$ is the volume element with respect to the metric $\langle$,$\rangle . Now, given A \subset D$, we set

$$
\mathfrak{H}(D ; A)=\left\{u \in H_{0}^{1}(D): \exists U \subset D \text { open, } A \subset U \text { and } u=1 \text { in } U\right\}
$$

and $H(D ; A)$ as the closure of $\mathfrak{H}(D ; A)$ with respect to $H^{1}$. With this notation we define the Capacity of $A$ as

$$
\operatorname{Cap} A=\inf \left\{\int_{D}|\nabla u|^{2} d M: u \in H(D ; A)\right\} .
$$

Below we have some well known consequences of this definition (see, e.g. [5], §4.7).

Proposition 2.1. For any $A \subset D$ we have,

i) $\operatorname{Cap} A=\inf \{\operatorname{Cap} U: U$ open, $A \subset U\}$.

ii) If $A_{1} \subset \cdots \subset A_{k} \subset \ldots$ are compact subsets of $D$, then

$$
\lim _{k \rightarrow \infty} \operatorname{Cap} A_{k}=\operatorname{Cap}\left(\bigcap_{k} A_{k}\right)
$$

We will use the notation $V^{\varepsilon}(A)$ to mean the tubular neighborhood of $A$ of radius $\varepsilon$, that is, $V^{\varepsilon}(A)=\{x \in M: \operatorname{dist}(x, A)<\varepsilon\}$, where dist (, ) stands for the distance function on $M$. Using this notation we have the following consequence of the above proposition:

Corollary 2.2. If $A$ is compact, then

$$
\lim _{\varepsilon \rightarrow \infty} \operatorname{Cap} V^{\varepsilon}(A)=\operatorname{Cap} A .
$$


In the case that $A$ is a submanifold of codimension $d \geq 2$ we can say more:

Proposition 2.3. If $A$ is an embedded submanifold of codimension $d \geq 2$, then there exists a family of open sets $V_{j} \supset A$ such that $\bigcap_{j} V_{j}=A$ and

$$
\text { Cap } V_{j} \rightarrow 0 \text { as } j \rightarrow \infty \text {. }
$$

In particular, Cap $A=0$.

This proposition follows from minor modifications of Theorem 3, page 154 of [5].

Now recall that $H_{0}^{1}(D)$ is a Hilbert space with respect to

$$
\left\langle u_{1}, u_{2}\right\rangle_{*}=\int_{D}\left\langle\nabla u_{1}, \nabla u_{2}\right\rangle d M
$$

In fact $\langle,\rangle_{*}$ and $\langle,\rangle_{H^{1}}$ yield equivalent norms, as we can see by using the Poincaré inequality

$$
|u|_{L^{2}(D)} \leq C|\nabla u|_{L^{2}(D)} .
$$

Hence $H(D ; A)$ is a closed (affine) subspace of $H_{0}^{1}(D)$ with respect to $\langle,\rangle_{*}$. Let $u_{A}$ be the orthogonal projection of 0 on $H(D ; A)$. By definition we get $\left|\nabla u_{A}\right|_{L^{2}(D)}^{2}=$ Cap $A$.

Now we start the proof of Theorem 1.2 following some ideas contained in [4]. Here we will consider nonempty boundary domains and general elliptic operators in the divergence form.

For each $x \in D$, let $P_{x}: T_{x} M \rightarrow T_{x} M$ be a symmetric, positive (or negative) definite operator. We define, for each $f \in C_{0}^{\infty}(D)$,

$$
L f=\operatorname{div}(P \nabla f)+q f,
$$

where $q: D \rightarrow \mathbb{R}$ is a bounded function.

We consider the unique extension of $L$ to $H_{0}^{1}(D)$. Then, $L$ is an elliptic operator, and let us denote by $\left\{\lambda_{0}<\lambda_{1} \leq \lambda_{2} \leq \ldots\right\}$ the spectrum of the Dirichlet problem of $L$ on $D$, repeated according to its multiplicity.

We recall Courant's min-max principle for the eigenvalues of $L$ on domains of $M$ :

$$
\lambda_{k}(D \backslash A)=\min _{E \in \mathcal{E}_{k}} \max _{f \in E \backslash\{0\}} \frac{\int_{D \backslash A}\left(\langle P \nabla f, \nabla f\rangle+q f^{2}\right) d M}{\int_{D \backslash A} f^{2} d M},
$$

where $\mathcal{E}_{k}$ is the set of $k$-dimensional subspaces of $H_{0}^{1}(D \backslash A)$. The quotient above is called the Rayleigh quotient for $f$.

In order to simplify some calculations below we set

$$
Q(f ; D):=\int_{D}\left(\langle P \nabla f, \nabla f\rangle+q f^{2}\right) d M .
$$

So, the Rayleigh quotient for $f$ in $D$ is given by $Q(f ; D) /|f|_{L^{2}(D)}^{2}$.

By using the analogous characterization for $\lambda_{k}(D)$ we easily obtain the first inequality of Theorem [1.2. In order to obtain the second inequality we choose $f_{1}, \ldots, f_{k}$, an orthonormal basis of eigenfunctions associated to $\lambda_{1}(D), \ldots, \lambda_{k}(D)$, and set $F_{k}$ the space generated by $f_{1}, \ldots, f_{k}$. Then $F_{k} \subset H_{0}^{1}(D)$ and

$$
\lambda_{k}(D)=\max _{f \in F_{k} \backslash\{0\}} \frac{Q(f ; D)}{|f|_{L^{2}(D)}^{2}} .
$$


We now define $E_{k}=\left\{g=f\left(1-u_{A}\right): f \in F_{k}\right\}$. It is clear that $E_{k} \subset H_{0}^{1}(D \backslash A)$ is a finite dimensional subspace. We will see that, when $A$ has small capacity, then $E_{k}$ has dimension equal to $k$. In fact, the functions $g_{j}=f_{j}\left(1-u_{A}\right), j=1, \ldots, k$, form a basis for $E_{k}$. We have that

$$
\left\langle g_{i}, g_{j}\right\rangle_{L^{2}(D)}=\delta_{i j}-2 \int_{D} f_{i} f_{j} u_{A} d M+\int_{D} f_{i} f_{j} u_{A}^{2} d M .
$$

Thus, using the Cauchy-Schwarz and Poincaré inequalities we have

$$
\begin{aligned}
& \left|\left\langle g_{i}, g_{j}\right\rangle_{L^{2}(D)}-\delta_{i j}\right| \leq 2\left|\int_{D} f_{i} f_{j} u_{A} d M\right|+\left|\int_{D} f_{i} f_{j} u_{A}^{2} d M\right| \\
& \leq 2\left(\int_{D}\left(f_{i} f_{j}\right)^{2} d M\right)^{\frac{1}{2}}\left(\int_{D} u_{A}^{2} d M\right)^{\frac{1}{2}}+\max _{D}\left(f_{i} f_{j}\right) \int_{D} u_{A}^{2} d M \\
& \leq C_{k}\left(\int_{D} u_{A}^{2} d M\right)^{\frac{1}{2}}+C_{k}^{\prime} \int_{D} u_{A}^{2} d M \\
& \leq B_{k}\left(\operatorname{Cap} A^{\frac{1}{2}}+\operatorname{Cap} A\right)
\end{aligned}
$$

where $B_{k}$ is a positive constant depending only on vol $D$ and $\max _{i=1, \ldots, k}\left|f_{i}\right|_{L^{\infty}(D)}$. On the other hand, we may choose $\varepsilon_{k}>0$ sufficiently small such that, if Cap $A<\varepsilon_{k}$, then

$$
B_{k}\left(\operatorname{Cap} A^{\frac{1}{2}}+\operatorname{Cap} A\right)<\min \left\{\left\langle g_{i}, g_{j}\right\rangle_{L^{2}(D)}-\delta_{i j}: i, j=1, \ldots, k\right\} .
$$

For such $\varepsilon_{k}$ we have that $g_{1}, \ldots, g_{k}$, form an orthonormal basis and consequently $E_{k}$ has dimension equal to $k$ as we claimed. Now we look for estimates of the numerator of the Rayleigh quotient for $g=f\left(1-u_{A}\right) \in E_{k}$, where $f \in F_{k}$ and $|f|_{L^{2}(D)}=1$. We first observe that for any closed subset $A \subset D$,

$$
|g|_{L^{2}(D)}^{2}=1-2 \int_{D} f^{2} u_{A} d M+\int_{D} f^{2} u_{A}^{2} d M \geq 1-B_{k}^{\prime} \text { Cap } A^{\frac{1}{2}}
$$

Now we have

$$
\begin{aligned}
& Q(g ; D)=\int_{D}\left(\left[\langle P \nabla f, \nabla f\rangle+q f^{2}\right]-2\langle P \nabla f, \nabla f\rangle u_{A}+\langle P \nabla f, \nabla f\rangle u_{A}^{2}\right) d M \\
& +\int_{D}\left(\left\langle P \nabla u_{A}, \nabla u_{A}\right\rangle f^{2}-2\left\langle P \nabla f, \nabla u_{A}\right\rangle f\left(1-u_{A}\right)-2 f^{2} u_{A}+f^{2} u_{A}^{2}\right) d M \\
& \leq \lambda_{k}(D)+B_{k}^{\prime \prime}\left(\operatorname{Cap} A+\operatorname{Cap} A^{1 / 2}\right),
\end{aligned}
$$

where $B_{k}^{\prime \prime}$ depends only on $\operatorname{vol} D, \max _{i=1, \ldots, k}\left|f_{i}\right|_{L^{\infty}(D)}, \max _{i=1, \ldots, k}\left|\nabla f_{i}\right|_{L^{\infty}(D)}$ and $\max _{D}\|P\|$.

Therefore, choosing $\varepsilon_{k}>0$ such that $|g|_{L^{2}(D)}^{2}>0$ in (2.1), we have

$$
\frac{Q(g ; D)}{|g|_{L^{2}(D)}^{2}} \leq \lambda_{k}(D)+\frac{\lambda_{k}(D) B_{k}^{\prime} \operatorname{Cap} A^{1 / 2}+B_{k}^{\prime \prime}\left(\operatorname{Cap} A+\operatorname{Cap} A^{1 / 2}\right)}{1-B_{k}^{\prime} \operatorname{Cap} A^{1 / 2}} .
$$

We observe that, if Cap $A<\varepsilon_{k}$, then we can estimate the second term above as

$$
\begin{aligned}
& \frac{\lambda_{k}(D) B_{k}^{\prime} \operatorname{Cap} A^{1 / 2}+B_{k}^{\prime \prime}\left(\operatorname{Cap} A+\operatorname{Cap} A^{1 / 2}\right)}{1-B_{k}^{\prime} \operatorname{Cap} A^{1 / 2}} \leq \frac{\lambda_{k}(D) B_{k}^{\prime} \operatorname{Cap} A^{1 / 2}}{1-B_{k}^{\prime} \varepsilon_{k}^{1 / 2}} \\
& +\frac{\left(B_{k}^{\prime \prime} \operatorname{Cap} A^{1 / 2}+B_{k}^{\prime \prime}\right) \operatorname{Cap} A^{1 / 2}}{1-B_{k}^{\prime} \varepsilon_{k}^{1 / 2}} \leq \frac{\left(\lambda_{k}(D) B_{k}^{\prime}+B_{k}^{\prime \prime} \varepsilon_{k}^{1 / 2}+B_{k}^{\prime \prime}\right) \operatorname{Cap} A^{1 / 2}}{1-B_{k}^{\prime} \varepsilon_{k}^{1 / 2}} \\
& =C_{k} \operatorname{Cap} A^{1 / 2} .
\end{aligned}
$$


So,

This implies that

$$
\frac{Q(g ; D)}{|g|_{L^{2}(D)}^{2}} \leq \lambda_{k}(D)+C_{k} \operatorname{Cap} A^{1 / 2}
$$

$$
\lambda_{k}(D \backslash A):=\max _{g \in E_{k} \backslash\{0\}} \frac{Q(g ; D)}{|g|_{L^{2}(D)}^{2}} \leq \lambda_{k}(D)+C_{k} \operatorname{Cap} A^{1 / 2},
$$

and we conclude the proof of Theorem 1.2

Corollary 2.4. Given a closed subset $A \subset D$ with Cap $A=0$, let $\left\{V_{j}\right\}$ be the family of open sets given in Proposition 2.3 and let $\lambda_{k}\left(D \backslash V_{j}\right)$ be the $k$-th eigenvalue of the Dirichlet problem of the operator $L$ in $D \backslash V_{j}$. Then

$$
\lambda_{k}\left(D \backslash V_{j}\right) \rightarrow \lambda_{k}(D) \quad \text { as } j \rightarrow \infty .
$$

In particular, if $\lambda_{k}\left(D \backslash V_{j}\right) \geq 0$ for all $j=1,2, \ldots$, then $\lambda_{k}(D) \geq 0$.

\section{Proof of Theorem 1.1}

We start by presenting an equivalent condition for $r$-stability. A simple computation shows that

$$
\operatorname{trace}\left(B^{2} P_{r}\right)=\left\|\sqrt{P_{r}} B\right\|^{2}:=\sum_{i=1}^{n} \theta_{i}^{2}(r) .
$$

On the other hand, Lemma 2.1 of [2] says that

$$
\operatorname{trace}\left(B^{2} P_{r}\right)=S_{1} S_{r+1}-(r+2) S_{r+2} .
$$

Thus, for $r$-minimal immersions, the Jacobi operator can be written as

$$
T_{r}=L_{r}+\operatorname{trace}\left(B^{2} P_{r}\right)=L_{r}+\left\|\sqrt{P_{r}} B\right\|^{2} .
$$

Using integration by parts, we have that

$$
\begin{aligned}
I_{r}(f, f) & =\int_{D}\left(\left\langle P_{r} \nabla f, \nabla f\right\rangle-\left\|\sqrt{P_{r}} B\right\|^{2} f^{2}\right) d M \\
& =\int_{D}\left(\left|\sqrt{P_{r}} \nabla f\right|^{2}-\left\|\sqrt{P_{r}} B\right\|^{2} f^{2}\right) d M .
\end{aligned}
$$

So, in the case $P_{r}>0$, to check that a regular domain $D \subset M$ is $r$-stable we just need to show that the last term above is always nonpositive or nonnegative for all $f \in C_{0}^{\infty}(D)$. Similarly in the case $P_{r}<0$.

Now we fix $\varepsilon>0$ and denote $D^{\varepsilon}=D \backslash V^{\varepsilon}(A)$. Then, the Gauss map $g$ is a local diffeomorphism on $D^{\varepsilon}$. Let $\varphi: g\left(D^{\varepsilon}\right) \rightarrow \mathbb{R}$ be the positive first eigenfunction of the spherical Laplacian $\widetilde{\Delta}$ on $g\left(D^{\varepsilon}\right)$, that is,

$$
\begin{cases}\widetilde{\Delta} \varphi+\lambda_{1}^{\varepsilon} \varphi=0 & \text { in } g\left(D^{\varepsilon}\right), \\ \varphi>0 & \text { in } g\left(D^{\varepsilon}\right), \\ \varphi=0 & \text { on } \partial g\left(D^{\varepsilon}\right),\end{cases}
$$

where $\lambda_{1}^{\varepsilon}=\lambda_{1}\left(D^{\varepsilon}\right)$ is the first eigenvalue of $\widetilde{\Delta}$ on $g\left(D^{\varepsilon}\right)$. Recall that, since $D^{\varepsilon} \subset D$, we have $\lambda_{1}^{\varepsilon} \geq \lambda_{1}$.

In the following we will consider the pull back metric $\widetilde{s}$ by $g$ on $D^{\varepsilon}$ and denote by $\widetilde{\nabla}$ the gradient, by [] the norm of a vector, and by $d S$ the volume element in this metric. By Lemma 2.9 in [1] one has $\widetilde{\nabla} f=B^{-2} \nabla f$, for smooth functions $f$ 
on $M$ and a simple computation gives $[X]=|B X|$, for any tangent vector $X$. Also we point out that, in the metric $\widetilde{s}$, the Gauss map $g: D^{\varepsilon} \rightarrow S^{n}$ is a local isometry. Now let $\psi=\varphi \circ g$ defined in $D^{\varepsilon}$. Then $\psi$ is positive and satisfies $\widetilde{\Delta} \psi+\lambda_{1}^{\varepsilon} \psi=0$ in $D^{\varepsilon}$. Thus, by Corollary 1 in $[\underline{6}$, we have that the first eigenfunction of the operator $\widetilde{\Delta}+\lambda_{1}^{\varepsilon}$ is nonnegative:

$$
0 \leq \inf \left\{\int_{D^{\varepsilon}}\left([\widetilde{\nabla} f]^{2}-\lambda_{1}^{\varepsilon} f^{2}\right) d S: f \in C_{0}^{\infty}\left(D^{\varepsilon}\right), \int_{D^{\varepsilon}} f^{2} d S=1\right\} .
$$

Now, since $\operatorname{det} g=S_{n}$ and the immersion is $r$-special on $D^{\varepsilon}$, we have $d S=$ $\left|S_{n}\right| d M=c\left\|\sqrt{P_{r}} B\right\|^{2} d M$, where $c$ is positive constant. Also, by hypothesis, $\lambda_{1}^{\varepsilon} \geq \lambda_{1} \geq \tau$. Thus

$$
\int_{D^{\varepsilon}}\left([\widetilde{\nabla} f]^{2}-\lambda_{1}^{\varepsilon} f^{2}\right) d S \leq c \int_{D^{\varepsilon}}\left([\widetilde{\nabla} f]^{2}-\tau f^{2}\right)\left\|\sqrt{P_{r}} B\right\|^{2} d M .
$$

Observe now that $\rho_{i}=\frac{\theta_{i}^{2}(r)}{\sum_{j} \theta_{j}^{2}(r)}, i=1, \ldots, n$, are the eigenvalues of the operator $\frac{\sqrt{P_{r}} B}{\left\|\sqrt{P_{r}} B\right\|}$. Therefore, by definition

$$
\tau:=\max _{i, D} \frac{1}{\rho_{i}^{2}}=\max _{D}\left|\left(\frac{\sqrt{P_{r}} B}{\left\|\sqrt{P_{r}} B\right\|}\right)^{-1}\right|^{2}
$$

So,

$$
[\widetilde{\nabla} f]^{2} \leq\left|\left(\frac{\sqrt{P_{r}} B}{\left\|\sqrt{P_{r}} B\right\|}\right)^{-1}\right|^{2}\left[\left(\frac{\sqrt{P_{r}} B \widetilde{\nabla} f}{\left\|\sqrt{P_{r}} B\right\|}\right)\right]^{2} \leq \tau\left[\left(\frac{\sqrt{P_{r}} B \widetilde{\nabla} f}{\left\|\sqrt{P_{r}} B\right\|}\right)\right]^{2} .
$$

Using this on (3.2), we have

$$
\begin{aligned}
c \int_{D^{\varepsilon}}\left([\widetilde{\nabla} f]^{2}-\tau f^{2}\right)\left\|\sqrt{P_{r}} B\right\|^{2} d M & \leq c \tau \int_{D^{\varepsilon}}\left(\frac{\left[\sqrt{P_{r}} B \widetilde{\nabla} f\right]^{2}}{\left\|\sqrt{P_{r}} B\right\|^{2}}-f^{2}\right)\left\|\sqrt{P_{r}} B\right\|^{2} d M \\
& =c \tau \int_{D^{\varepsilon}}\left(\left[\sqrt{P_{r}} B \widetilde{\nabla} f\right]^{2}-\left\|\sqrt{P_{r}} B\right\|^{2} f^{2}\right) d M .
\end{aligned}
$$

Now, we recall that $\widetilde{\nabla} f=B^{-2} \nabla f$ and $[X]=|B X|$ to find that

$$
\left[\sqrt{P_{r}} B \widetilde{\nabla} f\right]^{2}=\left|\sqrt{P_{r}} \nabla f\right|^{2} \text {. }
$$

We conclude that, for any $f \in C_{0}^{\infty}\left(D^{\varepsilon}\right)$ with $\int_{D^{\varepsilon}} f^{2} d S=1$,

$$
0 \leq c \tau \int_{D^{\varepsilon}}\left(\left|\sqrt{P_{r}} \nabla f\right|^{2}-\left\|\sqrt{P_{r}} B\right\|^{2} f^{2}\right) d M .
$$

This implies that the first eigenvalue of the Jacobi operator $T_{r}$ is nonnegative on $D^{\varepsilon}$ and thus, by Corollary 2.4, we have that the first eigenvalue of the Jacobi operator $T_{r}$ is nonnegative in all $D$. This shows that $D$ is $r$-stable.

Remark. In the case $P_{r}<0$ we may set $Q_{r}=-P_{r}$ in the above computations to conclude that

$$
0 \leq c \tau \int_{D^{\varepsilon}}\left(\left|\sqrt{Q_{r}} \nabla f\right|^{2}-\left\|\sqrt{Q_{r}} B\right\|^{2} f^{2}\right) d M .
$$

On the other hand the Jacobi operator is given by

$$
\begin{aligned}
T_{r} f & =\operatorname{div}\left(P_{r} \nabla f\right)+\operatorname{trace}\left(B^{2} P_{r}\right) \\
& =-\operatorname{div}\left(Q_{r} \nabla f\right)+\operatorname{trace}\left(B^{2} Q_{r}\right) .
\end{aligned}
$$


We conclude that the first eigenvalue of $T_{r}$ is nonpositive on $D$, and therefore we obtain $r$-stability according to our definition.

\section{ACKNOWLEDGMENTS}

The author would like to express his gratitude to Professor Manfredo P. do Carmo for suggestions and much encouragement and also wishes to thank Alexander Arbieto and Carlos Matheus for valuable discussions on the material of this paper. Finally, the author is thankful to IMPA-Rio de Janeiro and his staff.

\section{REFERENCES}

1. H. Alencar, M. do Carmo and M. F. Elbert, Stability of hypersurfaces with vanishing $r$ mean curvatures in Euclidean spaces. J. reine angew. Math. 554 (2003), 201-216. MR1952173 (2003k:53061)

2. J. L. M. Barbosa and A. G. Colares, Stability of hypersurfaces with constant $r$-mean curvature. Ann. Global Anal. Geom. 15 (1997), no. 3, 277-297. MR.1456513 (98h:53091)

3. J. L. M. Barbosa and M. P. do Carmo, On the size of a stable minimal surface in $R^{3}$. Amer. J. Math. 98(1976), 515-528. MR0413172 (54:1292)

4. G. Courtois, Spectrum of manifolds with holes. J. Funct. Anal. 134 (1995), no. 1, 194-221. MR.1359926 (97b:58142)

5. L. C. Evans and R. F. Gariepy, Measure theory and fine properties of functions. Studies in Advanced Mathematics (1992), 268 p. MR 1158660 (93f:28001)

6. D. Fischer-Colbrie and R. Schoen, The structure of complete stable minimal surfaces in 3manifolds of non-negative scalar curvatures. Comm. Pure Appl. Math. 33 (1980), 199-211. MR.562550(81i:53044)

7. J. Hounie and M. L. Leite, The maximum principle for hypersurfaces with vanishing curvature functions. J. Diff. Geom. 41 (1995), 247-258. MR.1331967 (96b:53080)

8. J. Rauch and M. Taylor, Potential and scattering theory on wildly perturbed domains, J. Funct. Anal. 18 (1975), 27-59. MR0377303 (51:13476)

Instituto de Matemática, Universidade Federal de Alagoas, Campus A. C. Simões, BR 104, Norte, Km 97, 57072-970, Maceió, AL, BraziL

E-mail address: petrucio@impa.br 